\title{
Origins of Intermodulation Distortion in A Pseudo-differential CMOS Beamforming Receiver
}

\author{
Negar Shabanzadeh \\ Faculty of Information Technology \\ and Electrical Engineering \\ University of Oulu \\ Oulu, Finland \\ negar.shabanzadeh@oulu.fi \\ Aarno Pärssinen \\ Faculty of Information Technology \\ and Electrical Engineering \\ University of Oulu \\ Oulu, Finland \\ aarno.parssinen@oulu.fi
}

\author{
Mahmoud Shehab \\ Nokia Oyj \\ Oulu, Finland \\ mahmudgoda@gmail.com
}

\author{
Rehman Akbar \\ Faculty of Information Technology \\ and Electrical Engineering \\ University of Oulu \\ Oulu, Finland \\ rehman.akbar@oulu.fi
}

\author{
Timo Rahkonen \\ Faculty of Information Technology \\ and Electrical Engineering \\ University of Oulu \\ Oulu, Finland \\ timo.rahkonen@oulu.fi
}

\begin{abstract}
This paper studies the generation mechanisms of nonlinear distortion in the combination of an inverter-based LNTA and a passive mixer. The dominant nonlinearity appears to be the quadratic $V_{g s} V_{d s}$ mixing term in the passive mixer, that first causes low-frequency IM2, and then up-converts it to IM3. It is found that adding a common-mode feedback cancels the IM2 in a pseudo-differential structure, and hence also reduces the IM3 caused by the cascaded second order nonlinearities significantly. The CMFB gain and bandwidth considerations and their effect on the results are also given, and it is concluded that from the linearity point of view, the feedback circuit does not have to be very wideband since the dominant distortion products originate from baseband.
\end{abstract}

Index Terms-nonlinearity analysis, Volterra series, distortion contribution, common-mode feedback

\section{INTRODUCTION}

The current trend of ever-increasing data rates has imposed the need for millimeter-wave range communication systems, which in turn require parallelism as a means of providing filtering, beam-steering, and most importantly keeping up with the data rate speed and improving the overall throughput. Although there has been a lot of interest in analyzing highly parallel systems in terms of linear parameters such as gain and phase, studying their nonlinearity has not received much attention yet [1] [2]. This paper focuses on the nonlinearity analysis of the circuit blocks in the IF section of a beamforming receiver architecture proposed in [2], [3]. The receiver is designed for $3 \mathrm{GHz}$, and is inductorless due to the multitude of channels. The simplified block diagram is shown in Fig. 1, consisting of inverter-based LNAs, passive mixers, phase control switches, and eventually a summing point in a transimpedance amplifier. The circuit structures for analysis have been taken from [3], but due to limitations in technology access, the device characteristics and comparisons

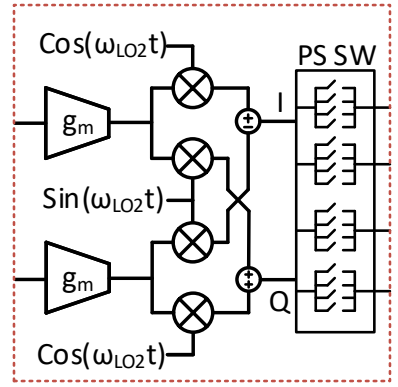

Fig. 1. Block diagram of one slice of the the beamforming receiver under analysis

to simulations have been performed using a different IC process. Hence, the performance is not an exact match to [3].

The nonlinearity analysis is based on fitting the transistor I-V characteristics (which are the most dominant cause of nonlinearity in active devices) to polynomials using the standard least square error method, and then utilizing the nonlinear current injection technique introduced by [4] and extended in [5] to illustrate the nonlinear contributions of each device as vector plots. In short, the analysis builds the small-signal model of the circuit, and adds the non-linear currents into it.

The purpose of this analysis was also to find out if there is a bias point where nonlinear coefficients are near to zero, or a pair of points that would lead to the distortions in two adjacent receiver slices canceling each other. For this purpose, the devices models were also fitted as functions of the bias point. 
The paper is organized as follows: Section II regards the nonlinearity analysis of a single transconductance stage or the low-noise amplifier part of the receiver. Section III includes the passive mixer into the analysis. The effect of the commonmode feedback is studied in parallel in sections II and III. CMFB gain and bandwidth considerations and their impacts on the results are investigated in Section IV. Finally, a discussion is given in Section V.

\section{TRANSCONDUCTANCE STAGE NONLINEARITY ANALYSIS}

The overall structure under analysis is shown in Fig. 2. M1M4 form the transconductance stage, M5-M8 are passive mixer transistors, and M9-M10 provide the common-mode feedback. We will start the analysis with the transconductance $(\mathrm{gm})$ stage, and add the subsequent stages step by step. A CMOS inverter with shunt feedback and rather low-ohmic load is used as a transconductance amplifier to transform input voltage into current. Working in current mode improves linearity by reducing the voltage swings, and makes it possible to perform gain control by summing up parallel branches. The circuit is dimensioned for a $1.5 \mathrm{~V}$ supply and $3 \mathrm{GHz}$ center frequency.

Calculating the distortion contributions is much easier if we model the device currents as polynomial functions of the controlling ac voltages, as in 1 :

$$
i_{d}=\sum K_{i j} v_{g s}^{i} v_{d s}^{j}
$$

Where $i$ and $j$ are the orders of the nonlinearity taken into account. The coefficients $K_{i j}$ can be found in several ways. Here, a simple least-square error fit has been used over a signal range of $\pm 200 \mathrm{mV}$ around the bias point. By moving the bias point, we can also see how the nonlinearity varies and choose the bias setup that could lead to minimal nonlinearity. Note that the transistor model in (1) can include both drain and gate voltage effects, and it is used for all the devices that are considered nonlinear.

As an example, Fig. 3 shows the input voltage related coefficients K10, K20, K30 of a CMOS inverter-based gm element in terms of the operating point. Curves are calculated for similar-shaped nMOS and pMOSes and at several input

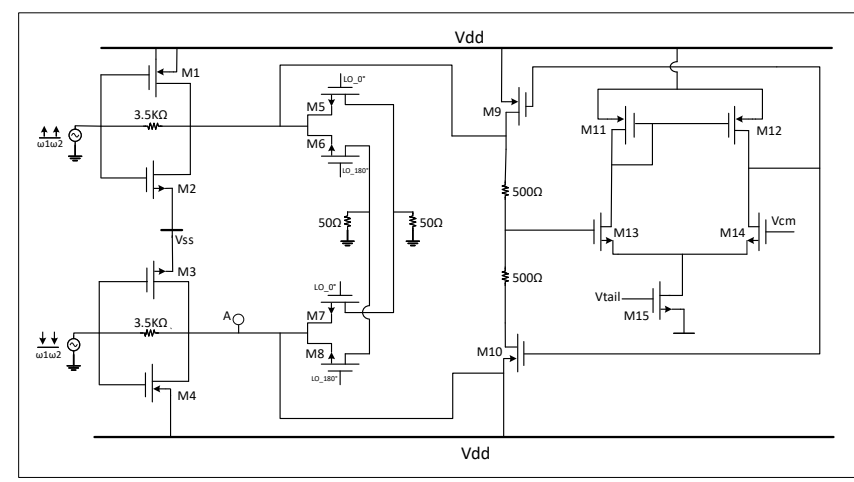

Fig. 2. Circuit schematic of the overall structure under analysis consisting of the differential gm element, passive mixers and the CMFB
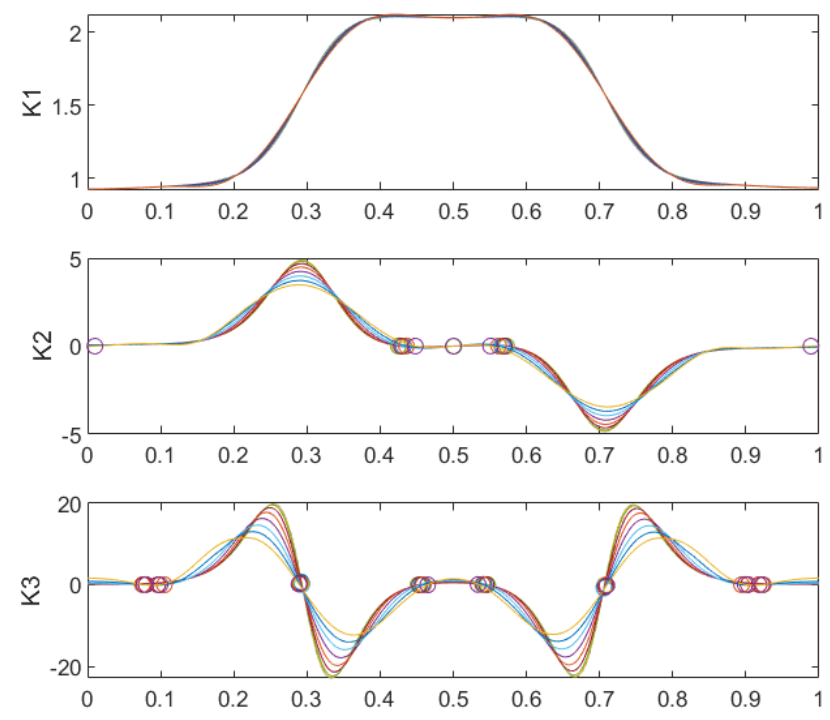

Fig. 3. Input-related nonlinearity coefficients vs. input bias point of a CMOS inverter gm stage

amplitude values (the peaks are flattened at larger amplitudes). It is seen that the nonlinearity is highest around the MOS threshold voltages, and minimized in the center. K1 (the linear gain) doubles when both devices are on, and $\mathrm{K} 2$ and $\mathrm{K} 3$ are small in the mid-bias range. Also, summing up the two transistors causes several minima in the nonlinearities (zerocrossings are marked with o's in the figure). Therefore, the bias can be chosen anywhere in the range Vin $=0.4 . .0 .6$, where either a distortion minimum or possible cancellations between two similar but differently biased branches can be achieved.

The simulations illustrate that the amplifier itself is very linear. A $10 \mathrm{mV}$ input was given in the two-tone test simulation setup, and the signal magnitudes at the gate and drain were $-49 \mathrm{dBV}$ and $-32 \mathrm{dBV}$ (all spectral measurements are from a two-sided spectra, so the values should be multiplied by two to get the amplitudes). The IM3 voltage in the output node was $-124.2 \mathrm{dBV}$. The use of common-mode feedback (CMFB) did not affect IM3, but it did reduce the IM2 from -80 to -89.8 $\mathrm{dBV}$. This is due to the fact that the even order nonlinearities appear as a common mode signal in differential structures, so the CMFB senses and attenuates them proportionally to the loop gain. In the case of the standalone gm, both IM3 and IM2 are already very low, and the effect of the CMFB will be more clear in the combination of a gm and mixer. It should also be stated that, although decreasing the feedback resistors improves the linearity, we would rather keep them maximized to avoid undesirable loading of the output signal of the gm stage. Therefore, a value of $500 \mathrm{ohms}$ has been chosen to address both these issues.

It is worth mentioning that the numerical values of improvements in nonlinearity are also very easily seen by traditional harmonic balance or pss simulation. However, the advantage 
of polynomial modeling is that it enables us to see which nonlinearity contribution is dominant, and how the inherent cancellation mechanisms are formed. This is illustrated by a vector plot where any higher order IM tone can be plotted, as shown in Fig. 4. Reading the vector plots requires a few considerations: The vector "Total" illustrates the magnitude and phase of the chosen IM tone similar to what we see in e.g. harmonic balance simulation, while the other vectors demonstrate how it is built from contributions from different nonlinearities. The contributions are labeled with their source device (e.g. gmm3 is the drain current response of transistor M3) and the degree of nonlinearity $\left(K_{i j}\right.$ refers to term $K_{i j} v_{g s}^{i} v_{d s}^{j}$ in the I-V equation (1)). Finally, the label indicates from which harmonic band the mixing is happening, such as baseband or second harmonic. The labels corresponding to the components smaller than a certain value have been removed for better readability.

According to Fig. 4, the cubic vgs-related nonlinearities (the K30 terms) of transistors M3 and M4 clearly dominate IM3 in the output node. There are also some cascaded secondorder mixing results (resulting from baseband (BB) or second harmonic (H2) band signals mixing to IM3). Although they are small in value, an interesting conclusion can be derived from the fact that the effects of nMOS and pMOS (gmm3k11O2BB and gm4k11O2BB) are cancelling each other. Since Early voltage is modeled with the $K_{11} v_{g s} v_{d s}$ term, this cancellation suggests that a low Early voltage does not degrade the linearity of an inverter stage, i.e. the inverter is not very sensitive to Early voltage variations.

Fig. 5 shows the results from pss analysis in Cadence Spectre corresponding to Fig. 4, giving an input-referred third-

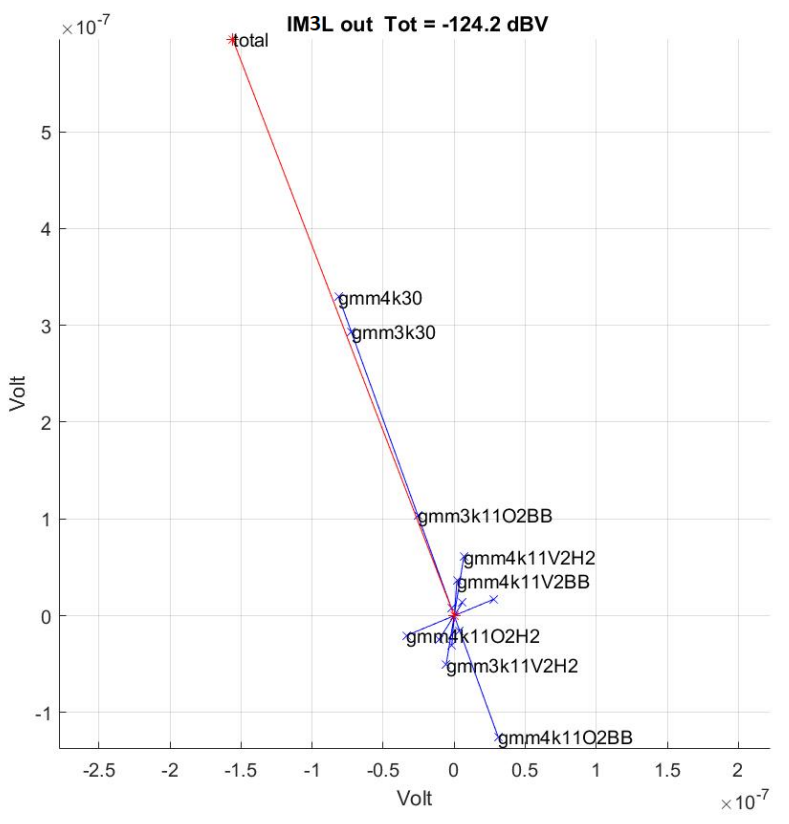

Fig. 4. Vector plots for IM3 voltage in the standalone differential gm stage

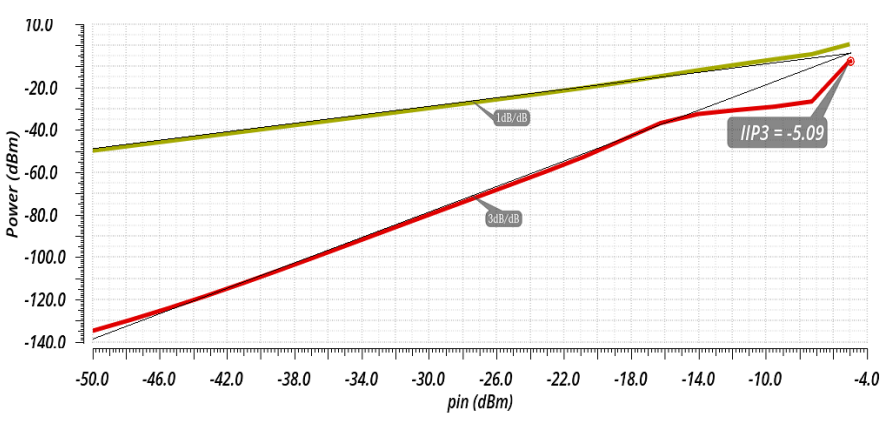

Fig. 5. IIP3 value of the gm-cmfb combination from Cadence pss simulation

order intercept point (IIP3) of $-5.09 \mathrm{~dB}$, which agrees with the value from the MATLAB simulations.

\section{MIXER STAGE NON-LINEARITY ANALYSIS}

The next stage to be taken into consideration is the mixer block. A passive mixer structure is commonly used due to its high linearity, high voltage headroom, low power consumption and good 1/f noise performance. We start the nonlinearity analysis at its simplest with a single transistor biased in the triode region and acting as a pass switch, which is continuously on during the analysis. The procedure resembles the one in [7], but is done numerically using the nonlinear current injection technique instead of closed-form kernel derivations. Also, we are including the effect of the common-mode feedback.

The DC fitting is done through the same procedure as described in section II, but now the device is in the triode region. Thus, the dominant nonlinearity in the nMOS switch is the fact that the signal voltage $v_{i n}$ directly modulates the gate-source voltage and on-resistance. Expanding the classical second order I-V equation $I_{D}=\beta\left(V_{G S}-V_{T}-V_{D S} / 2\right) V_{D S}$, we obtain an ac model of the form 2 :

$$
i=\beta V_{o n} v_{d s}-(\beta / 2) v_{d s}^{2}-\beta v_{d s} v_{i n}
$$

where the last term is usually dominant. Hence, a mixer introduces second order mixing in the conduction mode, which is not the desired RF mixing but a nonlinear effect that appears while the switch is conducting. The actual mixing could be modeled by extending the $V_{G S}$ fitting range beyond the threshold voltage and having sufficiently high order nonlinearity for the LO port. However, the current model is made by assuming that the mixer is constantly on (LO voltage is fixed), and then assuming the mixing as an ideal frequency translation. Although simplistic, this helps to study how IM3 is built up in the gm-mixer combination.

The first analysis case consists of a single gm and mixer without the common-mode feedback. As seen in Fig. 2, the simple single-transistor mixer has been extended to a doublebalanced structure now. The input is again a $10 \mathrm{mV}$ 2-tone, giving $-32 \mathrm{dBV}$ in the output of the gm stage. Now, the IM3 tones are some $40 \mathrm{~dB}$ higher than the case of a standalone gm element. To see what causes this, we need to look at the details of Fig. 6, which shows the IM3 contributions in the output of the gm element at node A. 


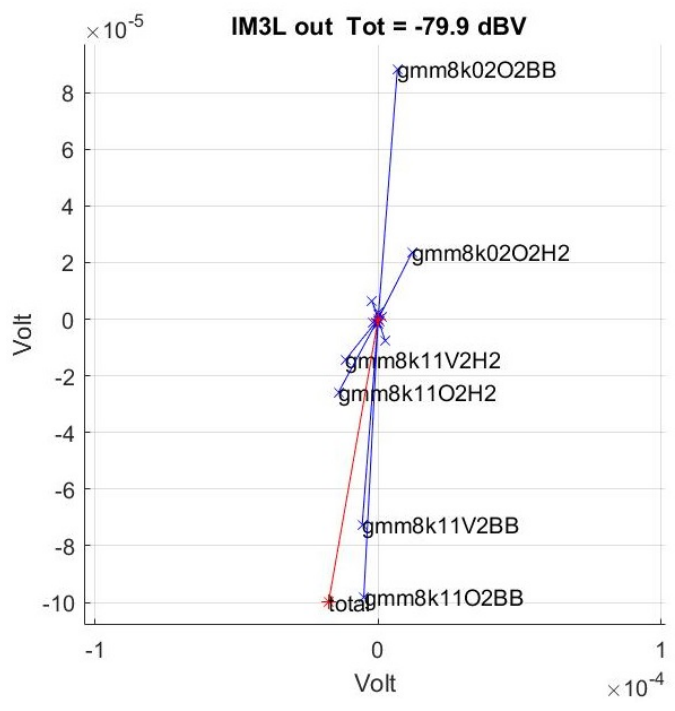

Fig. 6. Vector plot for IM3 output voltage in the gm-mixer combination without common-mode feedback

The vector plot shows all contributions from all the eight nonlinear devices M1-M8, and the dominant contribution seems to be the mixer transistor M8, where both the Ids curvature $\left(K_{02} v_{d s}^{2}\right)$ and the modulation of the on-resistance $\left(K_{11} v_{g s} v_{d s}\right)$ show strong but partially compensating contributions. These effects are again cascaded effects, generating IM2 voltages in the nodes around the mixer, and then mixing it further to IM3. Hence, these contributions can be reduced by filtering away the generated IM2 tones (baseband and second harmonic bands) at the drain and source terminals of the mixer transistor. Alternatively, as filtering consumes too much area, one can reduce the switch on resistance to minimize the $v_{d s}$ magnitude. For instance, [3] achieves this by employing LO bootstrapping to increase the gate drive.

The effect of the common-mode feedback is illustrated in Fig. 7, neglecting the nonlinearity of the CMFB transistors. Now, the feedback loop senses the generated IM2 and reduces its level from $-55 \mathrm{dBV}$ to $-79 \mathrm{dBV}$. Now that there is less signal to mix with the fundamental tones, the IM3 reduces from -80 to $-120 \mathrm{dBV}$. The quadratic effects are smaller in this case, and the cubic input-related nonlinearities of the gm transistors M3 and M4 (gmm3k30 and gmm4k30) are visible again in Fig. 7. Yet, the overall IM3 is still dominated by second-order mixing in M8 (terms gmm8k11OBB, gm8k11OH2), which is harvesting IM2 tones from both the baseband and second harmonic band.

Fig. 8 demonstrates the IIP3 result of the pss simulation corresponding to the gm-mixer combination with the commonmode feedback, which is again consistent with the MATLAB results.

\section{THE EFFECTS OF CMFB BANDWIDTH AND LINEARITY}

The choice of gain and bandwidth of the common-mode feedback circuit is the next thing to be considered. The $\mathrm{cm}$ correcting pMOS devices function as parallel loads for the

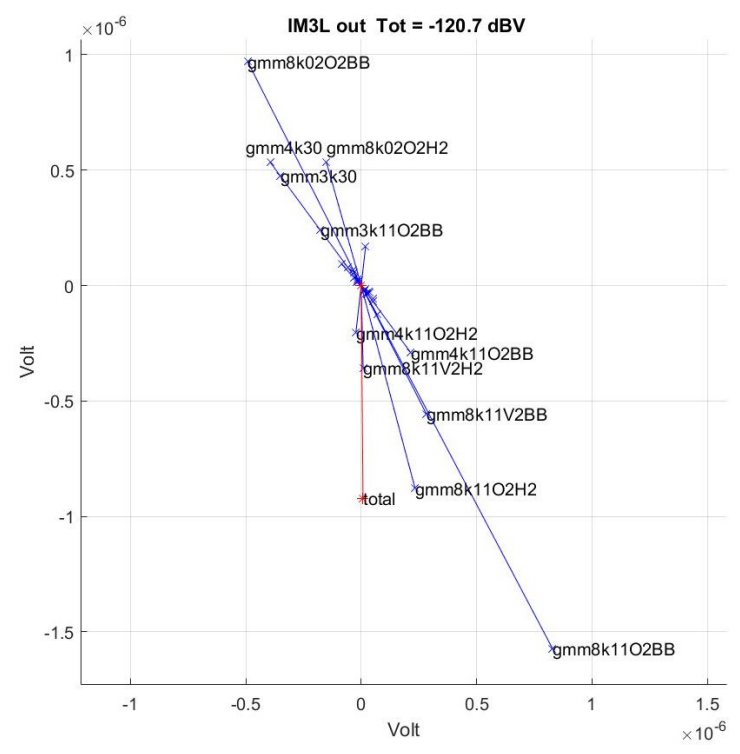

Fig. 7. Vector plots for IM3 nonlinear voltages in the gm-mixer combination with the common-mode feedback

cm signal, and to be effective, the total impedance they introduce should be noticeably smaller than the existing commonmode output impedance between the two transconductance and mixer stages. Keeping this in mind, we can either make the pMOSes smaller and compensate that by increasing the gain, and vice versa. There is not much difference between these two choices considering the linear signal only, although big devices change the biasing and loading of the inverter stage (which is why keeping them small is preferred). Nevertheless, the situation is more complex from distortion point of view. In addition to the linear common mode signal, any second order distortion in the amplifier and mixer is also seen as a common mode signal, and attenuating them is beneficial as seen above. Yet, there are two second order bands: the rectified signal near DC, and the second harmonic band which is difficult to attenuate due to its high frequency. This is where the bandwidth of the CMFB circuit comes into play.

From the linear signal point of view, the most narrowband solution is to correct just the dc offsets. However, if we aim at

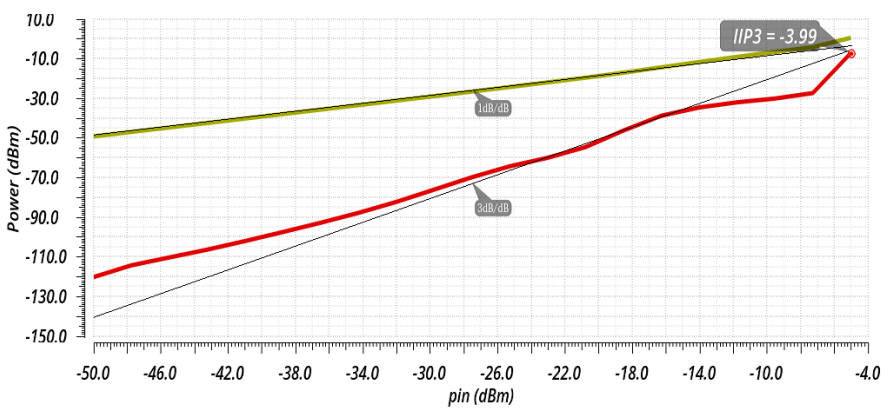

Fig. 8. IIP3 value of the gm-mixer-CMFB combination from Cadence pss simulation 
correcting amplitude mismatches e.g. due to a driving balun, the CMFB loop should have a bandwidth exceeding the carrier frequency. Therefore, we consider three different bandwidths which include only the beat frequency $(100 \mathrm{kHz})$, the carrier frequency $(3 \mathrm{GHz})$, and the second harmonic band $(6 \mathrm{GHz})$. The beat frequency is chosen arbitrarily, and as the main circuit in [3] supports a baseband bandwidth of over $400 \mathrm{MHz}$, it can also be pushed further. Results are presented in the Table 1 assuming both opposite-phased (case 1) and in-phase (case 2) inputs, and a third case where a $10 \%$ mismatch is introduced between the inputs. It is noteworthy that the differential input setup (case 1) is how the circuit is actually used, case 2 is only presented to illustrate common-mode rejection, and case 3 is an effort to simulate a more practical case, as presuming perfect symmetry in simulations hides some of the effects seen in reality. The op-amp gain is $25 \mathrm{~dB}$ in all examples, but since the gain is interdependent on the pMOS nonlinearities, we will get back to its effects once they are included.

TABLE I

THE EFFECT OF CMFB BANDWIDTH ON DISTORTION RESULTS

\begin{tabular}{|c|c|c|c|c|}
\hline Input Type & $\begin{array}{c}\text { CMFB } \\
\text { 3dB BW }\end{array}$ & $\begin{array}{c}\text { Linear Signal } \\
(\mathrm{dB})\end{array}$ & IM2 (dB) & IM3 (dB) \\
\hline case 1 & $100 \mathrm{kHz}$ & & -78 & -118.8 \\
& $3 \mathrm{GHz}$ & -33.4 & -77.7 & -121.3 \\
& $6 \mathrm{GHz}$ & & -77.7 & -121.3 \\
\hline case 2 & $100 \mathrm{kHz}$ & -32.4 & -75.4 & -112 \\
& $3 \mathrm{GHz}$ & -32.5 & -75.7 & -115.7 \\
& $6 \mathrm{GHz}$ & -32.8 & -76.3 & -117.7 \\
\hline case 3 & $100 \mathrm{kHz}$ & & -79.9 & -122.2 \\
& $3 \mathrm{GHz}$ & -34.4 & -79.5 & -124.6 \\
& $6 \mathrm{GHz}$ & & -79.5 & -124.4 \\
\hline
\end{tabular}

Table I suggests that increasing the CMFB bandwidth from $100 \mathrm{kHz}$ to $3 \mathrm{GHz}$ does not have a significant impact on the distortion cancellation, and the $3 \mathrm{GHz}$ and $6 \mathrm{GHz}$ cases are almost similar. To elaborate this, it is helpful to take another look at Figures 6 and 7: as seen in Fig. 6, the dominant cause of IM3 is the upconversion of the baseband IM2 result, while the second harmonic products are smaller and already cancelling each other to some extent. The same pattern is seen in Fig. 7 as well. So, even a narrowband CMFB can yield a considerable improvement in IM3. Although further increase in bandwidth helps to reduce the second order tones in the second harmonic band, it does not matter much if the bandwidth covers fundamental or second harmonics; the loop gain is sufficient in either case. This means that the CMFB circuit in this setup does not have to be very complex or capable of working at high frequencies to give satisfying results, as the improvement from Fig. 6 to Fig. 7 with the narrow-band op-amp CMFB in Fig. 2 also confirms.

The above discussion emphasizes the linearity of the feedback devices itself, as any nonlinearity originated in the feedback loop input is not attenuated by the loop. We have assumed completely linear devices in the CMFB loop in the previous sections, but it would be interesting to see if the nonlinearity of the controlling pMOSes has a significant impact on the results and how it can be relaxed by the choice of gain and bandwidth. Since the signal amplified by the loop drives the feedback devices, the combination of high gain and small devices (which was our design choice for the linear response) means that the nonlinearity will also be amplified. So, if this signal is large enough, the feedback loop must be strictly linear to maintain its positive effect. Luckily, the mentioned signal is not the input RF signal, but just the small common-mode error in it, in addition to small IM2 components. Hence, the controlling device's nonlinearity effect is mitigated to the point that it does not cause problems. The three cases given in Table I are simulated again with nonlinear feedback devices, and the results given in Table II are in agreement with this conclusion. As seen from Table II, IM3 increases by a maximum of 9 $\mathrm{dB}$ in the differential input and $14 \mathrm{~dB}$ in the mismatched input case, which is not slight yet low compared to the 30 $\mathrm{dB}$ cancellation we still have. The performance degradation is worse at higher frequencies and in case 3, which is also anticipated: the linearity of the feedback path matters if the feedback transistors can mix IM2 to IM3. This is illustrated if we have a mismatched condition where there is also a fundamental common-mode signal, as in case 3 . However, a $10 \%$ mismatch is still so small that the resulting deterioration in the total IM3 will not be significant (3-5 dB).

TABLE II

THE EFFECT OF CMFB NONLINEARITY ON DISTORTION RESULTS

\begin{tabular}{|c|c|c|c|c|}
\hline Input Type & $\begin{array}{c}\text { CMFB } \\
3 \mathrm{~dB} \mathrm{BW}\end{array}$ & $\begin{array}{c}\text { Linear Signal } \\
(\mathrm{dB})\end{array}$ & IM2 (dB) & IM3 (dB) \\
\hline case 1 & $100 \mathrm{kHz}$ & & -78 & -114.1 \\
& $3 \mathrm{GHz}$ & -33.4 & -77.7 & -112.8 \\
& $6 \mathrm{GHz}$ & & -77.7 & -112.7 \\
\hline case 2 & $100 \mathrm{kHz}$ & -32.4 & -75.4 & -107.8 \\
& $3 \mathrm{GHz}$ & -29.9 & -47.1 & -46.3 \\
& $6 \mathrm{GHz}$ & -25.4 & -44.3 & -42.5 \\
\hline case 3 & $100 \mathrm{kHz}$ & & -79.9 & -116.9 \\
& $3 \mathrm{GHz}$ & -34.4 & -80.3 & -112.1 \\
& $6 \mathrm{GHz}$ & & -80.2 & -110.3 \\
\hline
\end{tabular}

Finally, the effect of op-amp gain is studied and three cases with $15 \mathrm{~dB}, 25 \mathrm{~dB}$ and $35 \mathrm{~dB}$ gain are compared in Table III (the bandwidth is kept at $100 \mathrm{kHz}$ ). The linear signal and IM2 are fixed at $-33.4 \mathrm{~dB}$ and $-78 \mathrm{~dB}$, respectively. It is found that solely increasing the gain improves distortion cancellation, but this changes when the controlling pMOS sizes and nonlinear coefficients are scaled in proportion to the gain. (Note that the $25 \mathrm{~dB}$ gain case is taken as a standard, and the pMOSes in the other two cases are scaled with respect to it). In this case, the effect is positive from $15 \mathrm{~dB}$ to $25 \mathrm{~dB}$, but detrimental from $25 \mathrm{~dB}$ to $35 \mathrm{~dB}$. This is due to the fact that, as explained previously, the pMOS nonlinearities are amplified by this gain and could become effective past a certain gain value. We can conclude that sizing the pMOSes based on constant overall gm and choosing the gain accordingly is the best option, but increasing the gain further may make the distortion in the feedback loop visible. 
TABLE III

THE EFFECT OF CMFB OP-AMP GAIN ON DISTORTION RESULTS

\begin{tabular}{|c|c|c|}
\hline Op-amp Gain (dB) & IM3 (dB) & Scaled PMOSes \\
\hline 15 & -116.1 & No \\
\hline 15 & -113.2 & Yes \\
\hline 25 & -114.1 & - \\
\hline 35 & -111.5 & No \\
\hline 35 & -112.6 & Yes \\
\hline
\end{tabular}

\section{DISCUSSION}

This paper has investigated the mechanisms that generate IM3 in the combination of an inverter-type amplifier and a passive mixer. The utilized distortion contribution analysis technique allows to show the relative importance of all the contributions of polynomially modelled nonlinear devices.

The first finding was that, even though the inverter-type gm is quite linear, its linearity can still be further improved. Thus, a polynomial model of its input-output response was extracted at different bias points, and several zero-crossing points of the quadratic and cubic coefficients were observed in the operating band. Consequently, it was found that it is possible to bias the circuit in a sweet spot or near it, and even apply the parallelism of the structure so that if one gm slice is biased expansively, its neighbour would be compressive.

The second finding was that - as expected - the commonmode feedback loop attenuates the even-order nonlinearities that appear as common-mode interference in the output of the pseudo-differential amplifier. The amount of this attenuation depends on the available loop gain and bandwidth, which are also investigated in the paper.

The third finding was that, in the passive mixer, the input voltage directly modulates the $V_{G S}$ of the switch. This generates a strong second-order nonlinearity that first generates IM2 products, and then mixes them again with fundamental tones and generates IM3. The common-mode feedback is very useful here, as it reduces the IM2 level by $24 \mathrm{~dB}$ and IM3 level by 40 $\mathrm{dB}$, to a level where it can be said to match the performance of the gm amplifier again.

The fourth finding was that, luckily the bandwidth of the common-mode feedback does not need to be dramatically wide. With the baseband IM products being the most dominant cause of distortion, cancelling the low-frequency IM2 is already quite helpful. Also, as the first and second harmonic bands are just an octave away from each other, it does not matter much if the $3 \mathrm{~dB}$ bandwidth ends in the fundamental or the second harmonic band.

The fifth finding was that, the nonlinearity of the $\mathrm{cm}$ correcting pMOSes (which is assumed to be the dominant reason for nonlinearity regarding the CMFB) does not prove very destructive as the signal present in that node is small compared to the input RF signal. Although it causes 9-14 $\mathrm{dB}$ degradation in nonlinearity performance in the worst case scenario, there is still about $30 \mathrm{~dB}$ improvement over the nofeedback case. However, if the op-amp gain is too high, this effect may become more adverse.
Device I-V curves were extracted in Cadence, fitted to polynomials in Matlab, and calculated to vectors in Matlab using the NLSim program [8]. Results were checked against pss simulations in Cadence.

It should also be mentioned that so far, the analysis does not include the effects of switching, nonlinear capacitance or switching transients. This was motivated by simulating the complete receiver at two low and nominal LO frequencies without observing any noticeable difference in the total distortion. Nevertheless, these effects will definitely get important at higher frequencies, and could be inspiring for future works. The nonlinearity of the CMFB loop is also neglected, which could slightly affect the results as well.

\section{ACKNOWLEDGEMENT}

This project was funded by Finnish Academy project 325928 and Academy of Finland 6Genesis Flagship under Grant 318927.

\section{REFERENCES}

[1] N. Tervo, J. Aikio, T. Tuovinen, T. Rahkonen and A. Parssinen, "Digital predistortion of amplitude varying phased array utilising over-the-air combining," 2017 IEEE MTT-S International Microwave Symposium (IMS), Honololu, HI, 2017, pp. 1165-1168, doi: 10.1109/MWSYM.2017.8058809.

[2] M. C. M. Soer, E. A. M. Klumperink, D. van den Broek, B. Nauta and F. E. van Vliet, "Beamformer With Constant-Gm Vector Modulators and Its Spatial Intermodulation Distortion," in IEEE Journal of Solid-State Circuits, vol. 52, no. 3, pp. 735-746, March 2017, doi: 10.1109/JSSC.2016.2639545.

[3] R. Akbar et al., "A Wideband IF Receiver Module for Flexibly Scalable mmWave Beamforming Combining and Interference Cancellation," ESSCIRC 2019 - IEEE 45th European Solid State Circuits Conference (ESSCIRC), Cracow, Poland, 2019, pp. 213-216, doi: 10.1109/ESSCIRC.2019.8902869.

[4] Maas, Stephen A. Nonlinear Microwave and Rf Circuits. 2nd ed. Boston (Mass.): Artech House, 2003.

[5] J. P. Aikio and T. Rahkonen, "Detailed distortion analysis technique based on simulated large-signal voltage and current spectra," in IEEE Transactions on Microwave Theory and Techniques, vol. 53, no. 10, pp. 3057-3066, Oct. 2005, doi: 10.1109/TMTT.2005.855132.

[6] Liu and J. M. Rabaey, 'Linearity analysis of CMOS passive mixer," 2011 IEEE International Symposium of Circuits and Systems (ISCAS), Rio de Janeiro, 2011, pp. 2833-2836, doi: 10.1109/ISCAS.2011.5938195.

[7] H. Khatri, P. S. Gudem and L. E. Larson, "Distortion in Current Commutating Passive CMOS Downconversion Mixers," in IEEE Transactions on Microwave Theory and Techniques, vol. 57, no. 11, pp. 2671-2681, Nov. 2009, doi: 10.1109/TMTT.2009.2031930.

[8] A. Heiskanen and T. Rahkonen, "5th order electro-thermal multi-tone Volterra simulator with component-level output," Proceedings of the 2003 International Symposium on Circuits and Systems, 2003. ISCAS '03., Bangkok, 2003, pp. IV-IV, doi: 10.1109/ISCAS.2003.1206156. 